\title{
Impact of Laser Phase Noise on Nonlinear Frequency Division Multiplexing Systems
}

\author{
Da Ros, Francesco; Gaiarin, Simone; Zibar, Darko
}

Published in:

Proceedings of 2019 Conference on Lasers and Electro-Optics

Link to article, DOI:

10.1364/CLEO_SI.2019.SW3O.6

Publication date:

2019

Document Version

Peer reviewed version

Link back to DTU Orbit

Citation (APA):

Da Ros, F., Gaiarin, S., \& Zibar, D. (2019). Impact of Laser Phase Noise on Nonlinear Frequency Division Multiplexing Systems. In Proceedings of 2019 Conference on Lasers and Electro-Optics [8749214] IEEE. 2019 Conference on Lasers and Electro-Optics, CLEO 2019 - Proceedings https://doi.org/10.1364/CLEO_SI.2019.SW3O.6

\section{General rights}

Copyright and moral rights for the publications made accessible in the public portal are retained by the authors and/or other copyright owners and it is a condition of accessing publications that users recognise and abide by the legal requirements associated with these rights.

- Users may download and print one copy of any publication from the public portal for the purpose of private study or research.

- You may not further distribute the material or use it for any profit-making activity or commercial gain

- You may freely distribute the URL identifying the publication in the public portal 


\title{
Impact of Laser Phase Noise on Nonlinear Frequency Division Multiplexing Systems
}

\author{
Francesco Da Ros, Simone Gaiarin, and Darko Zibar \\ DTU Fotonik, Technical University of Denmark,DK-2800 Kongens Lyngby, Denmark,.fdro@fotonik.dtu.dk
}

\begin{abstract}
The impact of Wiener phase noise on NFDM transmission is experimentally investigated for dual-polarization discrete NFDM systems. The results show minimal OSNR penalty in back-to-back and limited degradation for $2000-\mathrm{km}$ transmission for $750-\mathrm{kHz}$ and $100-$ $\mathrm{kHz}$ linewidth, respectively.

OCIS codes: $060.1660,060.4510$
\end{abstract}

\section{Introduction}

In order to combat the signal quality degradation due to the onset of fiber nonlinearity, optical fiber transmission based on nonlinear frequency division multiplexing (NFDM) has been proposed [1]. The concept of NFDM is rooted in the theory of the nonlinear Fourier transform (NFT), i.e. the inverse scattering transform. The NFT allows solving the nonlinear Schrödinger equation in absence of fiber loss and third order dispersion, i.e. when the equation becomes integrable. By using such mathematical tools, signaling schemes constructively including the impact of fiber nonlinearity can be designed. Whereas promising results have been reported, with experimental demonstrations focusing on data transmission encoding the information on either the discrete spectrum (solitonic component) [2-5], the continuous spectrum (dispersive component) [6], or even both simultaneously [7], a comprehensive investigation of the impact of component limitations both at the transmitter- and receiver-side is still desirable. Even though experimental demonstrations are forced to deal with system limitations such as the impact of fiber loss and noise from optical amplifiers, most of the investigations rely on using narrow-linewidth lasers $(<\mathrm{few} \mathrm{kHz})$, often in homodyne configuration at the receiver side. Only a few preliminary analysis of the impact of laser phase noise on the performance of NFDM transmission have been reported. In particular, in [5] the performance degradation, stronger than for conventional transmission systems, is discussed for modulation of the continuous spectrum. The impact of phase noise on the discrete spectrum is also studied by characterizing the variance of the received spectral amplitudes [3] and eigenvalues [8] as the transmitter linewidth is varied.

In this work, we investigate the topic further by considering a two-eigenvalue dual-polarization NFDM transmission system with QPSK modulation operating at $250 \mathrm{MBd}(2 \mathrm{~Gb} / \mathrm{s})$. The impact of transmitter phase noise on the BER is experimentally evaluated in back-to-back configuration with receiver-side noise loading and for up to $2000 \mathrm{~km}$ of transmission.

2. Experimental setup

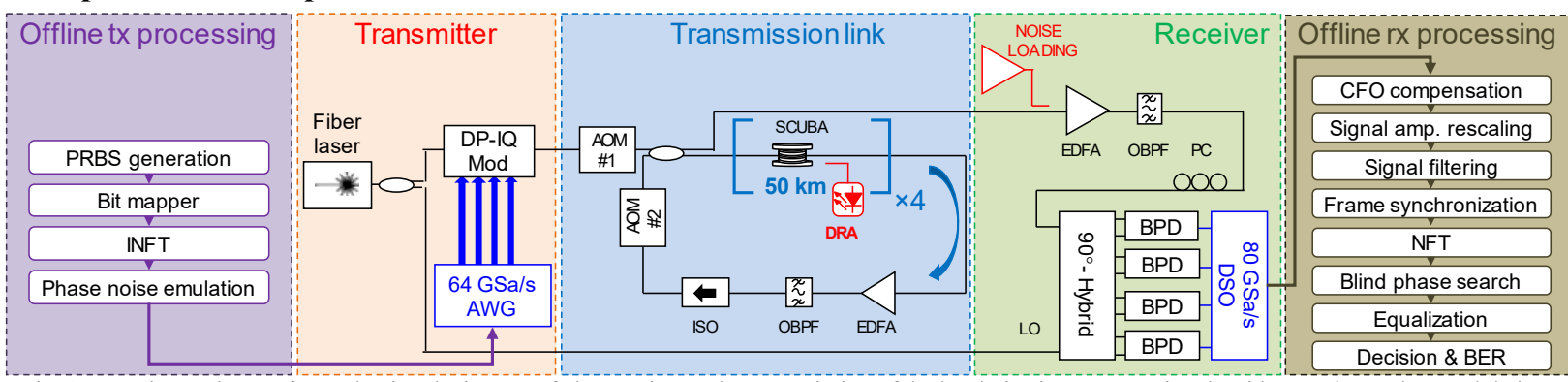

Fig. 1. Experimental setup for evaluating the impact of phase noise on the transmission of dual-polarization NFDM signals with two-eigenvalue modulation.

Fig. 1 shows the experimental setup. Pseudo random bit sequences (PRBSs) are generated and mapped to the dualpolarization $\mathbf{b}\left(\lambda_{\mathrm{i}}\right)$-scattering coefficients of a two-eigenvalue $\left(\lambda_{\mathrm{i}}=\{0.3 \mathrm{i}, 0.6 \mathrm{i}\}\right)$ NFDM signal. Only modulation of the discrete spectrum is considered and the modulation formats of choice for the $\mathbf{b}\left(\lambda_{\mathrm{i}}\right)$ are QPSK and $\pi / 4$-shift QPSK for the $\lambda_{1}$ and $\lambda_{2}$, respectively as in [3]. An inverse NFT (INFT) operation provides the time-domain waveform from the $\mathbf{b}\left(\lambda_{i}\right)$. Digitally emulated Weiner phase noise with the desired linewidth is then added to the signal before digitally-to-analog (D/A) converting it in a 64-GSa/s arbitrary waveform generator (AWG) and encoding it onto an optical carrier by a dual-polarization IQ modulator. The optical carrier originates from a narrow-linewidth (sub kHz, correlation length $>2000 \mathrm{~km}$ ) laser, so that the main contribution to the transmitter phase noise comes from the digital phase noise emulation. The 250 -MBd optical signal is injected into a recirculating transmission loop based on 
low-loss submarine fiber (SCUBA fiber) and distributed Raman amplification (DRA) with backward pumping every $50-\mathrm{km}$ to minimize the power variations along the link [4]. The recirculation loop includes an erbium-doped fiber amplifier (EDFA), an optical bandpass filter and acusto-optic modulators (AOMs) acting as loop switches [4]. The baud rate was limited by the available launch power into the loop and the necessity to fulfill the soliton conditions.

After a number $(N \geq 0)$ of re-circulations, the signal is received by a pre-amplified coherent receiver using as local oscillator (LO) the transmitter laser to avoid additional phase noise at the receiver side. After analog to digital conversion, offline digital signal processing (DSP) is applied. The DSP chain consists of static carrier frequency offset (CFO) compensation to remove the fixed frequency shift introduced by the AOMs, low-pass filtering, and frame synchronization to align the symbol slots for the NFT operation. The NFT operation recovers the eigenvalues and $\mathbf{b}$-coefficients from the time-domain waveforms. Then, phase noise compensation using standard blind phase search (BPS) with optimized parameter is applied independently on the four b-coefficients and NFT-domain equalization using a least-mean square algorithm $[4,5]$ is performed before bit-error rate (BER) counting.

Two scenarios are considered: back-to-back case with no transmission $(N=0)$ and additional noise loading at the receiver input (noise loading stage in Fig. 1); transmission case $(N=\{32,40\})$ with no receiver side noise loading.

\section{Experimental results}

The measured BER is reported in Fig. 2. Fig. 2(a) shows the back-to-back case, where the BER as a function of the received optical signal-to-noise ratio (OSNR) is shown for several emulated laser linewidths. The performance indeed degrades with the laser linewidth, resulting in error floors, but only for rather large linewidth values above $750 \mathrm{kHz}$ $\left(\Delta v \times T_{s}=0.003\right)$. Fig 2(b), instead, reports the performance at two transmission distances as the transmitter linewidth is varied. The impact of phase noise is stronger than in the back-to-back case but limited degradation can be seen for up to $100 \mathrm{kHz}$ of linewidth. Compared to conventional systems, in NFDM additional phase noise is generated during transmission by fiber loss which result in a displacement of the eigenvalues. Such a displacement leads to a rotation of the constellation [4,5], thus explaining the stronger impact of phase noise for the transmission scenario. Note that, in our investigation, the baud rate was limited; therefore moving to higher baud rates is expected to further relax the requirements on the transmitter laser linewidth. Additionally more refined phase noise tracking schemes than blind phase search, especially if customized for NFDM systems, could further reduce the degradation.

(a)

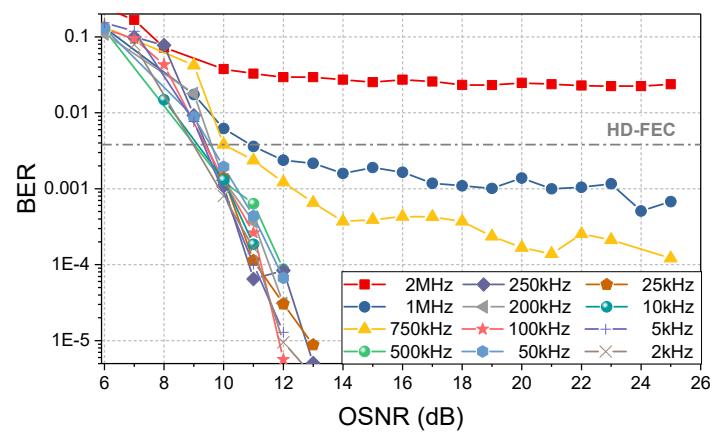

(b)

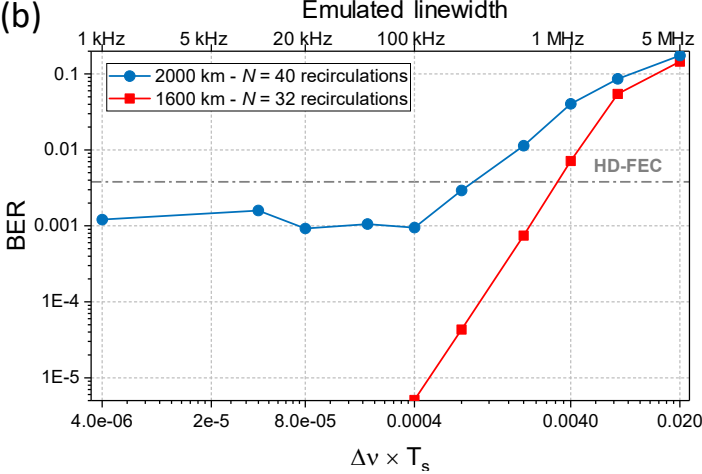

Fig. 2. (a) BER as a function of the received OSNR for different transmitter linewidths (back-to-back scenario) and (b) BER as a function of the transmitter linewidth for 1600-km and 2000-km transmission distances (transmission scenario)

\section{Conclusions}

We investigated the impact of transmitter phase noise on the BER performance on dual-polarization NFDM systems. The results show that even for low baudrate signals $(250 \mathrm{MBd})$, the penalty from the transmitter phase noise is limited for linewidths up to $750 \mathrm{kHz}$ (back-to-back) and $100 \mathrm{kHz}$ (transmission).

\section{Acknowledgements}

This work is supported by the European Research Council (ERC-CoG FRECOM project, 771878). We thank OFS Denmark for the SCUBA fiber.

\section{References}

[1] M.I. Yousefi and F.R. Kschischang, "Information transmission using the nonlinear Fourier transform, part I: mathematical tools," IEEE Trans. Inf. Theory 60, 4312 (2014).

[2] S. Gaiarin, et al., "Dual-polarization nonlinear Fourier transform-based optical communication system," Optica 5, 263 (2017).

[3] T. Gui, et al., "Phase Modulation on Nonlinear Discrete Spectrum for Nonlinear Frequency Division Multiplexed Transmissions," OFC 2016, W3A.2.

[4] S. Gaiarin, et al., "Dual-polarization NFDM transmission using distributed Raman amplification and NFT-domain equalization," PTL 30, 1983 (2018).

[5] T. Gui, et al., "Alternative Decoding Methods for Optical Communications Based on Nonlinear Fourier Transform,” JLT 35, 1542 (2017).

[6] S.T. Le, et al., "Equalization-Enhanced Phase Noise in Nonlinear Inverse Synthesis Transmissions,” ECOC 2016 , pp. 370.

[7] S.T. Le, et al., "Nonlinear signal multiplexing for communication beyond the Kerr nonlinearity limit," Nat. Phot., 11, 570 (2017).

[8] Z. Dong, et al., "Impact of Frequency Offset and Laser Phase Noise on Nonlinear Frequency Division Multiplexed Systems via the Nonlinear

Fourier Transform," ACP 2015, ASu3F.2. 